\title{
Acute Disseminated Encephalomyelitis Following 2009 H1N1 Influenza Vaccination
}

\author{
Kengo Maeda and Ryo Idehara
}

\begin{abstract}
Since the worldwide spread of the novel influenza type A virus in 2009, trivalent vaccines against H1N1 (pandemic) 09 and seasonal influenza have been used. We describe a 33-year-old woman who presented with hypoesthesia below the Th7 level fifteen days after vaccination without any preceding infection. Cerebrospinal fluid showed an increased level of myelin basic protein and positive oligoclonal $\operatorname{IgG}$ bands. Magnetic resonance imaging revealed disseminated lesions in the brain and thoracic cord. Steroid therapy improved her symptoms. She was diagnosed as having acute disseminated encephalomyelitis (ADEM) possibly related to the vaccination. As a potential adverse effect of the influenza vaccine, in addition to Guillain-Barré syndrome, ADEM should also be recognized.
\end{abstract}

Key words: acute disseminated encephalomyelitis (ADEM), influenza vaccine, H1N1, pandemic

(Intern Med 51: 1931-1933, 2012)

(DOI: 10.2169/internalmedicine.51.7487)

\section{Introduction}

In 2009, a novel influenza type A virus (H1N1 pandemic 09) emerged in Mexico and the United States, and spread rapidly worldwide $(1,2)$. In Japan, the first case of H1N1 09 infection was reported in May of that year. Since 2010, trivalent vaccines for $\mathrm{A} / \mathrm{H} 1 \mathrm{~N} 109, \mathrm{~A} / \mathrm{H} 3 \mathrm{~N} 2$, and $\mathrm{B}$ have been widely used for the prevention of influenza infection. Influenza vaccination, except for the 1976 swine influenza vaccine (A/New Jersey/76 H1N1), has been shown not to be significantly associated with neurological adverse effects (3). Before the pandemic, although acute disseminated encephalomyelitis (ADEM) following seasonal influenza vaccination had been thought to be very rare, a few cases were reported $(4,5)$. Since it is important to collect information about the safety of the vaccine for H1N1 09 influenza, we herein describe a patient who contracted ADEM following trivalent influenza vaccination including vaccine for H1N1 09.

\section{Case Report}

A 33-year-old woman received trivalent inactivated influ- enza vaccine (A/California/7/2009 (H1N1) pdm09, A/Victoria/210/2009 (H3N2), B/Bribane/60/2008; Denka Seiken Co.) the 9th of November 2011. In 2010 she had received an influenza vaccine for the first time. There was no adverse reaction at the time of vaccination. After fifteen days, she experienced numbness of her legs, and the numbness had ascended to the hip level by the next day. An orthopedist recommended that she came to our hospital. On examination, she was alert and could walk by herself. She did not complain of headache, nausea, or fever. Her cranial nerves and the muscle strength of all her limbs were normal. There was hyperesthesia at the Th7 dermatome on both sides. She reported hypoesthesia of tactile sensation below that level. Vibration and position sensation were normal. Deep tendon reflexes were normally evoked without pathological reflexes. There was no cerebellar or sensory ataxia. She did not have complication with severe constipation or urinary retention. Counts of blood cells, hepatic and renal function, electrolytes, blood glucose, and lipid metabolism were all normal. Thyroid function was also normal. C-reactive protein level was within the normal limit. Anti-nuclear antibody, anti-SS$\mathrm{A}$, and anti-SS-B antibody were negative. There was no pleocytosis in the cerebrospinal fluid (CSF). The levels of protein, glucose, and chloride of CSF were normal. How-

Department of Neurology, National Hospital Organization Shiga Hospital, Japan

Received for publication February 7, 2012; Accepted for publication April 4, 2012

Correspondence to Dr. Kengo Maeda, maeda-kengo@shiga-hosp.jp 


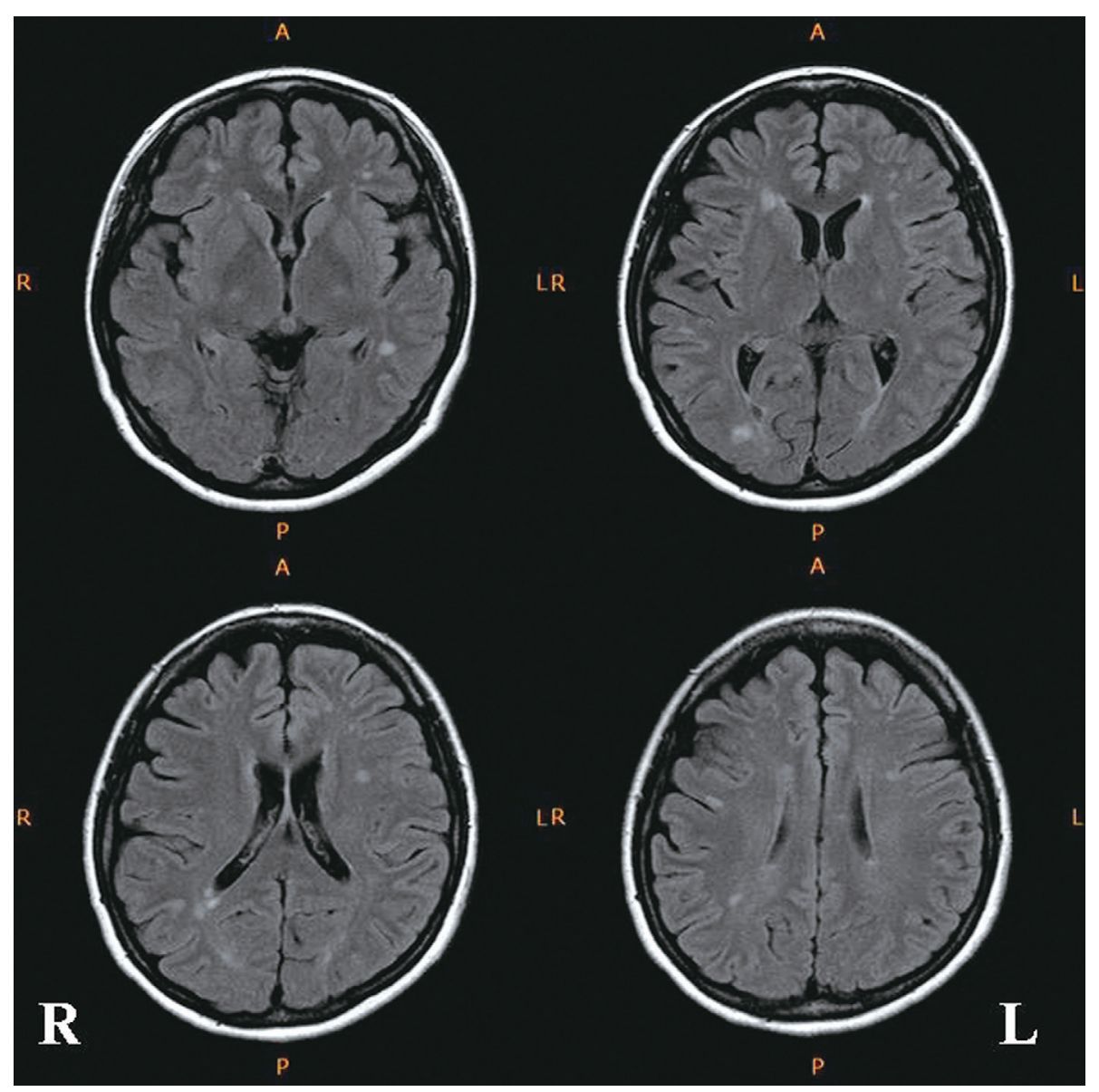

Figure 2. Scattered hyperintense lesions were found in the white matter of the cerebral hemispheres on FLAIR images of MRI.

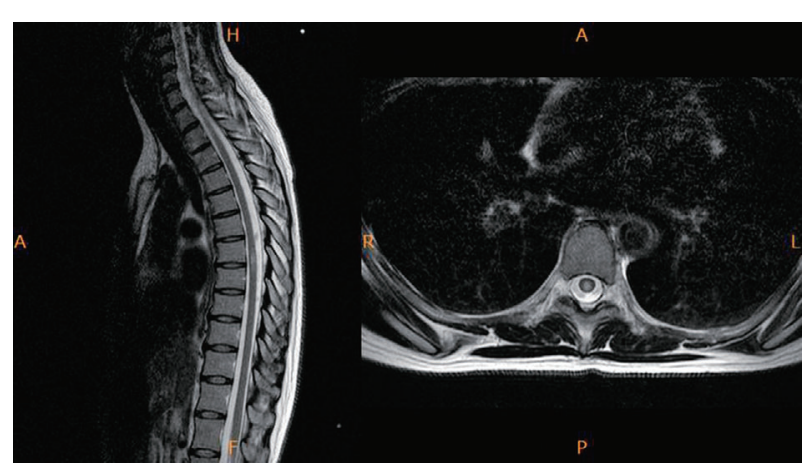

Figure 1. Hyperintense lesion was found in the thoracic cord on T2-weighted MRI.

ever, myelin basic protein was elevated to $128 \mathrm{pg} / \mathrm{mL}$ (normally $<102 \mathrm{pg} / \mathrm{mL}$ ), and oligoclonal IgG bands were positive. IgG index was 0.75 . T2-weighted magnetic resonance imaging (MRI) revealed a hyperintense lesion in the thoracic spinal cord (Fig. 1). There was no lesion in the cervical or lumbosacral cord. On FLAIR images of MRI, scattered hyperintense lesions were also found in the white matter of the cerebral hemispheres (Fig. 2). There was no abnormality in the visual acuity, visual field, or visual evoked potentials. Since there was no preceding infection, she was diagnosed as having ADEM associated with influenza vaccination. Her symptoms could have resulted from the involvement of the spinothalamic tracts. She was treated with intravenous infusion of methylprednisolone (1 g/day for 3 days). The level of CSF myelin basic protein was decreased to $60.5 \mathrm{pg} / \mathrm{mL}$ after steroid pulse therapy. Subsequently, prednisolone (30 $\mathrm{mg}$ /day) was administrated orally, and gradually tapered, during the following month. Her numbness was limited to her toes by two weeks after initiation of oral steroid therapy, and she noted numbness only at night during the next two weeks. The follow-up MRI performed two months later revealed resolution of the thoracic cord lesion.

\section{Discussion}

This patient was diagnosed as having ADEM. According to a flow diagram depicting modified WHO causality assessment, her neurological event was considered to be "possibly" related to influenza vaccination (6). Among the reports of the neurological adverse effects associated with H1N1 vaccines occurring between October 1, 2009 and March 31, 2010 (6), Guillain-Barré syndrome (GBS) was the most frequent (79 cases; $37.3 \%$ of the total neurological adverse effects). ADEM was also reported to occur (8 cases; $3.8 \%$ ). Four of the eight reported cases of ADEM were assessed to be "possibly" related to the vaccines. The mean age and 
mean interval to onset of these four cases were 20.7 years and 19.8 days, respectively. The vaccine types used were trivalent inactivated influenza vaccine in two cases, liveattenuated influenza vaccine in one case, and unknown in one case. In the English literature, we found six case reports of ADEM (7-10) and one case report of transverse myelitis (11). In Japan after the pandemic, 13 cases of ADEM after influenza vaccination were reported between November 1, 2010 and May 31, 2011 (12). Seven of these cases were considered by specialists to be undeniably as an adverse effect of the vaccination. In the same report, GBS associated with vaccination was reported to have occurred in 10 cases. Although much of the attention has been directed to GBS, we should also be aware of demyelinating diseases of the central nervous system that may develop after 2009 H1N1 influenza vaccination.

Post-vaccination ADEM has already been reported in association with several vaccines. As to the influenza vaccine, Nakayama and Onoda reported three cases of ADEM among those who received 38.02 million doses of influenza vaccine administered between 1994 and 2004 in a study by the Kitasato Institute in Japan (13). Case reports of ADEM associated with influenza vaccine are very rare $(4,5)$. Although it is difficult to establish a causal relationship between ADEM and a component of the trivalent influenza vaccine, the occurrence of ADEM seems to have become more frequent after the pandemic than before the pandemic. Large population studies regarding the adverse effects of influenza vaccines containing 2009 H1N1 influenza vaccine are desirable.

The authors state that they have no Conflict of Interest (COI).

\section{References}

1. Butler D. Portrait of a year-old pandemic. Nature 464: 1112-1113, 2010.

2. CDC. The 2009 H1N1 pandemic: summary highlights, April 2009-2010, a virus emerges. Available from: http://www.cdc.gov/ h1n1flu/cdcresponse.htm\#virus.emerges; 2010.

3. Kaplan JE, Katona P, Hurwitz ES, Schonberger LB. Guilain-Barré syndrome in the United States, 1979-1980 and 1980-1981: lack of an association with influenza vaccination. JAMA 248: 698-700, 1982.

4. Nakamura N, Nokura K, Zettsu T, et al. Neurologic complications associated with influenza vaccination: two adult cases. Intern Med 42: 191-194, 2003.

5. Shoamanesh A, Traboulsee A. Acute disseminated encephalomyelitis following influenza vaccination. Vaccine 29: 8182-8185, 2011.

6. Williams SE, Pahud BA, Vellozzi C, et al. Causality assessment of serious neurologic adverse events following 2009 H1N1 vaccination. Vaccine 29: 8302-8308, 2011.

7. Denholm JT, Neal A, Yan B, et al. Acute encephalomyelitis syndromes associated with H1N1 09 influenza vaccination. Neurology 75: 2246-2248, 2010.

8. Lapphra K, Huh L, Scheifele W. Adverse neurologic reactions after both doses of pandemic H1N1 influenza vaccine with optic neuritis and demyelination. Pediatr Infect Dis J 30: 84-86, 2011.

9. Fernandes AF, Marchiori PE. Bithalamic compromise in acute disseminated encephalomyelitis following H1N1 influenza vaccine. Arq Neuropsiquiatr 69: 571, 2011.

10. Lee ST, Choe YJ, Moon WJ, Choi JW, Lee R. An adverse event following $2009 \mathrm{H} 1 \mathrm{~N} 1$ influenza vaccination: a case of acute disseminated encephalomyelitis. Korean J Pediatr 54: 422-424, 2011.

11. Arcondo MF, Wachs A, Zylberman M. Transverse myelitis associated with anti-influenza A (H1N1) vaccination. Medicina (B Aires) 71: 161-164, 2011.

12. Ministry of Health, Labour and Welfare. Available from: http:// www.mhlw.go.jp/stf/shingi/2r9852000001 jqmw.html (in Japanese).

13. Nakayama T, Onoda K. Vaccine adverse events reported in postmarketing study of the Kitasato Institute from 1994 to 2004 . Vaccine 25: 570-576, 2007.

(C) 2012 The Japanese Society of Internal Medicine http://www.naika.or.jp/imindex.html 